\title{
Computational Models of Natural Language Argument
}

\author{
Chris Reed $^{1}$ and Floriana Grasso ${ }^{2}$ \\ 1 Department of Applied Computing, University of Dundee, Scotland \\ chris@computing.dundee.ac.uk \\ 2 Department of Computer Science, University of Liverpool, England \\ floriana@csc.liv.ac.uk
}

\begin{abstract}
This paper offers an introduction to the 2001 Workshop on Computational Models of Natural Language Argument (CMNLA 2001), a special event of the International Conference on Computational Science. The contributors to the workshop represent, in their backgrounds, the diversity of fields upon which the focus of the event draws. As a result, this paper aims not only to introduce the accepted papers, but also to provide a background that will be accessible to researchers in the various fields, and to sit each work into a coherent context.
\end{abstract}

\section{Introduction}

There is rapidly growing interest in the applications of argumentation for computer reasoning. One of the most mature and substantial research projects in the area is Pollock's OSCAR system [36, but the use of argumentation and dialectics in computational contexts has become increasingly popular, and with a variety of aims. Emphasis has been put on many aspects of the argumentation process: from the study on the structure of "valid" arguments 1326, to the way complex arguments can be unreeled 948. Argumentation is starting to play a key role in communication in multi-agent systems with significant results reported, inter alia, in 32. Perhaps not by coincidence, argumentation theory itself has been enjoying a renaissance in recent years, demonstrated by the wide and extensive bibliography offered by 43 .

Surprisingly, the ways in which arguments are rendered in natural language, the most obvious vehicle for the purpose, have not been investigated at depth, apart from few exceptions [10,28,31,37]. The endeavour is particularly challenging, both for computational linguists and for computer scientists, as argumentation is typically rich with rhetorical devices interacting at many different layers of abstraction, and is heavily dependent upon extra-linguistic context if it is to be successful. Moreover, a vast literature on both argumentation theory and rhetoric can offer great potential for exploitation, and, as in many other computational modelling problems, cross-disciplinary fertilisation is crucial for achieving significant results.

The 2001 Workshop on Computational Models of Natural Language Argument (CMNLA 2001) aims exactly at bringing this issue into the limelight. 
The emphasis of the workshop is therefore squarely on computational models of natural language argumentation. As a result, the introduction and discussion presented here avoids the rich literature and fertile areas of research in which formal models of argumentation are employed for artificial reasoning, and computer-computer communication. Similarly, topics within the venerable field of argumentation theory in its own right do not here form a key focus of study, although it is inevitable that discussion at the workshop itself should draw on these fields. The discussion will instead focus on framing the problem, by providing a background that will be accessible to researchers in the various fields. The paper also aims to introduce the accepted papers, and, by emphasising the diversity of fields upon which the focus of the event draws, to sit each work into a coherent context.

\subsection{Computational Models of Discourse}

Computational Linguistics scientists concentrate on the "study of computer systems for understanding and generating natural language" [20], in order to develop "a computational theory of language, using the notions of algorithms and data structures from Computer Science" [1]. Typical problems for these research fields are [1]: How is the structure of sentences identified? How can knowledge and reasoning be modelled? How can language be used to accomplish specific tasks? Research spans, naturally enough, across the two ends of the human communication problem: understanding natural language, and producing it.

Understanding a piece of text is not merely a problem of "parsing" the sentences it comprises to identify the grammatical structure that can describe it. It is not even, or not only, to extract the "meaning" of such sentences, taking care of inherent natural language ambiguities. It is recognised that discourse has a much more complex structure than the simple collection of its sentences, and higher level factors must come into play in deciding whether a text is coherent. In other words, understanding text means primarily establishing whether such text had a purpose, in the mind of the writer, and how the purpose has been achieved by the writer, with the choice of one particular articulation of the sentences. Enlightening examples of such "rhetorical" parsing are in 29]. To achieve this level of comprehension, works on discourse annotation become of paramount importance, as they allow to gain a deeper understanding of how this presumed "structure" of a text is build up by human writers (a remarkable example for argumentative texts is [41]).

The same assumption that text has a structure, that can be determined, at least partially, by the writer's goal, has driven research in automatic natural language generation, for the complementary task of producing a piece of text in a human language from computer accessible, or, in general, non-linguistic representation of information [40. If the text has to be produced for a purpose, and not by just gluing together sentences, knowledge is required of, at least, the domain of discourse, the purpose of the piece of text, the strategy to achieve such purpose, the rules for discourse organization, and, in order for the text to be tailored to a particular audience, the characteristics of the addressee. 
Models of the structure of discourse are based on diverse hypotheses. For example, many of the early natural language processing systems have associated discourse with the concept of "recipe", or schema: if most of the texts containing an explanation (e.g. in an encyclopedia) have the same structure (start with the identification, "X is a $\mathrm{Y}$ ", then pass to listing peculiar attributes of $\mathrm{X}$ etc.) then we can ascribe the same structure to all texts having the same purpose (to explain) [33. Other systems are based on a different, less rigid approach: following Austin's intuition that utterances are performatives just as physical actions are [3, the metaphor of a "plan" has been used to describe discourse [2]22]. Just as a robot may have the goal of building a brick tower, and can decompose this problem into smaller and smaller tasks, until easily executable basic actions can be performed (lift arm, pick up block etc.), similarly a natural language tool may decompose a communicative goal into its steps, and use a plan based mechanism to achieve them. The original problem is then transformed into the problem of deciding how such communicative goals can be defined in the first place, and how they can be achieved, that is decomposed into smaller, more manageable problems. Guidance on these issues comes typically from discourse organisation theories, the most popular of which is perhaps the Rhetorical Structure Theory (RST) [27], a theory which, despite some criticisms [34], has been very widely used (see for example [24]).

\subsection{Computational Models of Argumentative Discourse}

The emerging area across computational linguistic and argumentation theory sets itself the task of investigating the forms and varieties in which arguments are put forward in natural language. Important issues are, therefore, those concerning the linguistic characteristics of argumentative texts: discourse markers, sentence format, referring expressions, style, and in general how natural language arguments can be produced on one hand, and recognised on the other. But equally fundamental are considerations on models of the argumentative process itself, in terms of characteristics of the audience [35], ultimate judge of the quality of an argument: their beliefs and propensities, but also emotions, personalities, values, norms. Preliminary reflections on this subject are included in [17]. The CMNLA 2001 workshop aimed at establishing common grounds for research, by gathering together researchers from very diverse and complementary fields, from computer science, to linguistic, to philosophy. Together, the contributors worked at defining the gaps that need to be bridged, while at the same time proposing avenues to bridge them.

\section{The Gap at the Human Computer Interface}

Work at the human-computer interface is confounded by two related challenges. The first arises as a result of human limitations, and the need to restrict, abridge, and limit, whilst at once explaining, repeating, and emphasising information in such a way that it can be successfully assimilated by the human. Computational 
models of interaction, and particularly linguistic models of interaction, must necessarily take account of the limitations of the human interlocutor if they are to succeed. This consideration becomes all the more important in more demanding communicative situations, where, for example, the aim is to effect attitude change through persuasion, or to offer explanation through the expression of complex reasoning.

The second challenge is posed by the limitations of the communicating computer system, or, more accurately perhaps, by the traditional design of such systems. A sound and productive engineering approach has led to the development of natural language processing systems which build incrementally, one on the next. This much is to be valued most highly, and is taken as an indicator of a growing maturity in the field. It has also inevitably led to the widespread adoption of simplifying assumptions and a specific circumscription of the problem which are only now starting to be questioned. The underlying representation of linguistic and extra-linguistic knowledge, and the tasks which should properly be considered as part of the domain of Natural Language Processing are thus becoming important items on the research agenda in their own right.

Together, these two challenges represent a gap at the human-computer interface, comprising both the oversight of human limitations, and the underestimation of factors that need to be accounted for. Computational implementations of argumentation are one means of closing this gap. Theories of argument have squarely addressed complex communicative encounters involving persuasion and explanation, and offer insight into ways of circumventing, handling, and even exploiting, human limitations apposite in such situations. Furthermore, argumentation theory has, necessarily, embraced a much wider range of issues that impinge on communication. Similarly, computational systems focused specifically upon argumentation are necessitating a broader view of what constitutes Natural Language Processing, and are demanding reconsideration of some of the basic assumptions of the field.

\subsection{Human Limitations}

Following complex argumentation is an extremely demanding task for most people. If the single, simple deductive step embodied in the Wason selection task [45] can unerringly produce such dismal performance - and its manifold variations produce such wide discrepancies - then it should come as no surprise that comprehending substantial chains of argumentative steps represents a superbly intensive cognitive task. Even in cases in which the structure of argumentation is quite straightforward, the job of assimilating the data presented is not a simple one. Fox et al.'s [12] work on computer systems which reason and then present explanations in the oncology domain has clearly demonstrated that whilst formal models of argumentation provide a powerful mechanism for reasoning under conditions of uncertainty, the subsequent presentation of that reasoning to a human is highly troublesome.

In the first place, there are two conflicting forces which introduce a tension into the process of generating text involving arguments. On the one hand, 
humans tire easily, becoming bored if information is repeated, or even if inferences are laboriously drawn out explicitly. This is reason for the ubiquity of enthymemes, in which components of an argument step are left implicit. Instead of carefully enumerating conclusion and minor and major premises of a Modus Ponens (as in The litmus paper turned red, and if litmus paper turns red then the solution is acid, so the solution is acid), one might more naturally offer an enthymematic version (The litmus paper turned red, so the solution is acid).

On the other hand, humans also have a tendency to get lost in an argument, and typically require a battery of linguistic devices to mark the way. A prime example is offered in the use of repetition and confirmation in informationally redundant utterances or IRUs, described by Walker [44]. IRUs have a range of functions including aid memory, support saliency, etc. but what concerns us here is the very idea that it is necessary at all to introduce components which convey no new information. Reed's [37] analysis of similar phenomena takes a related, if symmetrically opposite, approach whereby such utterances are not added to a text, but are instead always present at a deep level, and pruned as appropriate at the surface level - this analysis then supports explanation of differences in occurrences of enthymemes. For although enthymematic contraction is almost canonical in arguments structured around Modus Ponens, it is extremely rare in Modus Tollens constructions.

This tension between the need for brevity on the one hand and the requirements of waymarking on the other is one of the key issues addressed in Fiedler and Horacek [11. The domain for their P. Rex system is the presentation of logical proofs. It is quite clear from their analysis that even in the relatively stylised linguistic usage of mathematical proof, it is crucial to be able to determine when an enthymeme (or other generic form of argument contraction) is appropriate, and when it is not. To render a proof comprehensible, information must be omitted where obvious or easily inferred (or where it occurs in particular configurations of particular syllogisms), but explained in detail where necessary.

The waymarking which is so vital to argument comprehension is perhaps best exemplified in the use of discourse markers (also, cues or clues). The use of specific lexical items marking argument structure was first discussed in the computational linguistics literature by Cohen [7, and has since played an important role in several models of natural language argument generation including [1018, 38]. Carenini's GEA system [5], however, is one of very few which follows the task of generating the text of an argument from high level intentional goal through to textual output. Here, after selecting argument content, various components contribute to the ultimate lexical realisation, including processing to introduce appropriate discourse markers, aimed at making the reader's task that much easier - and the aims of the argument consequently that much more likely to be fulfilled.

In addition to these opposing forces working on the level of detail in an argument, and the introduction of discourse markers, there are also further considerations of human limitations and susceptibilities available to computer systems engaging argument. One is the technique of 'loading' or 'affect' - using biased or 
evaluative terms to more or less subtly influence the reception of the argument. Hovy [23] included a study of affect in his PAULINE system; by altering values for a small range of stylistic variables (formality, force, etc.) the structure and lexical choice effected by the system could be radically altered. Gavenko's discussion [15] offers a broad analysis of particular forms of such affect including the use of visual and haptic metaphor. A computational model armed with the ability to flexibly employ such metaphors would significantly enhance the argumentation that could be generated by aiding the interlocutor in their task of comprehension.

\subsection{Computer Limitations}

To many in computational linguistics, and particularly in projects concentrating on argumentation, sentences like "The CN Tower is the world's tallest free standing structure", or, "The CN Tower is an engineering marvel", are typically represented in some knowledge base as p, or, q. Both are simply examples of 'propositions'. Gilbert [16] performs two tasks. The first is to remind us of the fact that such an abstraction is woefully inappropriate. Where the first sentence is (in the right context) a fact, the other (in the right context) is a value; the former does not admit of argumentation, whilst the latter does. Gilbert then discusses how the distinction between, and arrangement of, such facts and values is dependent upon the 'field' of context. Each user then represents not just one field, but a prioritized hierarchical arrangement of many different fields. This characterisation is similar to the trees of preferences used in systems such as 18 and [5], but Gilbert's suggestion goes further by associating with a given user not just a network of preferences (and values, and beliefs), but rather, a whole host of such networks, one for each role that the user might adopt. These networks are then themselves arranged in a hierarchy depending upon the context. Issues of inheritance and conflict between fields are going to play an important role in any system adopting this view of users, and these interactions are as yet ill-understood. There are, however, promising directions such as the application of context logic for capturing the closely related notion, due to Perelman and Ohlbrechts-Tyteca [35], of 'audience'. Preliminary work on this idea is to be reported in 8].

Perhaps the strongest tradition in widening the remit of natural language processing systems is in the incorporation of various non-textual information. One of the key pieces of work in the area is Maybury's system of 'communicative acts' which he applied both to the generation of argument 31 and to the inclusion of multimedia sources [30]. Green's analysis [19], examines how multimedia resources are employed in real argumentation, in an attempt to enrich automated generation of multimedia-rich argument. The structure of argumentation in non-textual source is notoriously difficult to identify in anything but the most trivial of examples, but recent work has made advances in this direction. Groarke's study [21], in particular, shows how art and editorial cartooning can be analysed to reveal deductive structure, and how that structure can then be assessed. 
A third traditional restriction in the design of natural language processing systems is founded on intuitions about ethics and the role of the computer. Machines have not traditionally been equipped with the ability to deceive. And yet, it is becoming clear that in particular scenarios, such a capability is crucial. In negotiating (with one user, on behalf of another), or dealing with emotive issues (the oncological domain of Fox et al. 12 mentioned above is a rich source) there is a demand in the former case, for deception, and in the latter, for at least some equivocation. Recognition of the importance of the role of insincerity is what has motivated the work of Carofiglio and de Rosis [6], who build a probabilistic account based on Toulmin [42] structures of argument. Of course, as Carofiglio and de Rosis point out, deception carries with it a risk of discovery, which could be damaging in any communicative encounter. An analysis of cases of human deception in 4 demonstrates that equivocation plays a key role in salving the conscience and offering means of recovery in the event of discovery. The subtleties of linguistic prevarication employed in deception are beyond most current computational systems which typically exploit knowledge of a user's misconceptions (such as the 'licentious' flag in Zukerman et al.'s [4] NAG system). The analysis offered by Carofiglio and de Rosis provides a means to start refining the mechanisms which can be employed in the various forms of insincere communication.

Finally, a crucial challenge to be overcome if computers are to engage in dialogic argument is the problem of comprehension. Natural language understanding itself is an immense challenge of course, but understanding argument poses further problems above and beyond understanding the individual discourse elements. In order to understand and, ultimately, be in a position to be able to respond to human argument, it is essential that the large scale structure which provides the scaffolding is recognised accurately. This, after all, is one of the primary aims of the (primarily North American) undergraduate courses on argument analysis supported by texts such as 46. The prospect of being able to automatically produce the sorts of diagrams available in, for example, Freeman's [14] analyses is extremely attractive. But to achieve that sort of competence, there are substantial problems upon which work has barely begun: automatic argument reconstruction, fallacy detection, the recognition of argument forms, and so on.

\section{Prospects}

The overlap between argumentation theory and Artificial Intelligence (AI) is particularly lucky as it can draw upon talented researchers in an extremely wide array of fields: linguistics, psychology, mathematics, cognitive science, philosophy, rhetoric, speech communication, politics, computer science, law, social science, economics, and more. The substantial work that can be achieved when members of these fields converge on argumentation was demonstrated dramatically at the Symposium on Argument and Computation, held in Scottish Highlands in June 2000. During the course of a week, two dozen scholars, most of whom 
had not met previously, worked together to write a book examining the areas of interdisciplinary overlap. The remarkable results, which are to be published as [39], testify not only to the dedication of the contributors, but also to the level of interest that is being sustained in the area.

With a foundation like this, the prospects for argumentation and AI are extremely good - but there is a great deal of work to be done. Events like the Symposium on Argument and Computation and CMNLA are not only indicators of the interest within both fields in the crossdisciplinary overlap, but are also catalysts in stimulating new collaborations. The fruits of these collaborations hold very great promise.

\section{Acknowledgements}

We are most grateful to our colleagues who acted as reviewing committee, thus providing immense help in achieving a stimulating final programme:

- Cristiano Castelfranchi, Department of Communication Science, University of Siena, Italy.

- Fiorella de Rosis, Department of Informatics, University of Bari, Italy.

- Leo Groarke, Department of Philosophy, Wilfrid Laurier University, Waterloo, Ontario, Canada.

- Ehud Reiter, Department of Computer Science, University of Aberdeen, Scotland.

- Antoinette Renouf, Department of English Language and Literature, University of Liverpool, England.

We are also grateful to SIGGEN, the Association for Computational Linguistics Special Interest Group in Natural Language Generation, for kindly offering support-in-name to the workshop.

\section{References}

[1] J.F. Allen. Natural Language Understanding. The Benjamin/Cummings Publishing Company, Inc., 2nd edition, 1995.

[2] D.E. Appelt. Planning English Sentences. Studies in Natural Language Processing. Cambridge University Press, 1985.

[3] J. Austin. How To Do Things With Words. Oxford University Press, 1975.

[4] J.B. Bavelas, A. Black, N. Chovil, and J. Mullet. Truth, lies and equivocation. Journal of Language and Social Psychology, 9(1-2):135-161, 1990.

[5] G. Carenini. GEA: a Complete, Modular System for Generating Evaluative Arguments. In (current volume).

[6] V. Carofiglio and F. de Rosis. Exploiting Uncertainty and Incomplete Knowledge in Deceptive Argumentation. In (current volume).

[7] R. Cohen. Analyzing the Structure of Argumentative Discourse. Computational Linguistics, 13(1-2):11-24, 1987.

[8] J. Crosswhite, J. Fox, C.A. Reed, T. Scaltsas, and S. Stumpf. Computational Models of Rhetoric. In Reed et al. 39, (to appear). 
[9] H. Dalianis and P. Johannesson. Explaining Conceptual Models - Using Toulmin's argumentation model and RST. In Proceedings of The Third International workshop on the Language Action Perspective on Communication Modelling (LAP98), pages 131-140, 1998.

[10] M. Elhadad. Using Argumentation in Text Generation. Journal of Pragmatics, 24:189-220, 1995.

[11] A. Fiedler and H. Horacek. Argumentation in Explanations to Logical Problems. In (current volume).

[12] J. Fox and S. Das. A unified framework for hypothetical and practical reasoning (2): lessons from medical applications. In D. Gabbay and H.J. Olbach, editors, Practical Reasoning: Proceedings of FAPR'96. Springer-Verlag, 1996.

[13] J. Fox, P. Krause, and M. Elvang-Goransson. Argumentation as a General Framework for Uncertain Reasoning. In D. Heckerman and A. Mamdani, editors, Proceedings of the 9th Conference on Uncertainty in Artificial Intelligence, pages 428-434. Morgan Kaufmann Publishers, 1993.

[14] J.B. Freeman, editor. Dialectics and the MacroStructure of Argument. Foris, 1991.

[15] S. Gavenko. Analysis of the Argumentative Effect of Evaluative Semantics in Natural Language. In (current volume).

[16] M.A. Gilbert. Getting Good Value: Facts, Values and Goals in Computational Linguistics. In (current volume).

[17] M.A. Gilbert, F. Grasso, L. Groarke, C. Gurr, and J.M. Gerlofs. The Persuasion Machine: An Exercise in Argumentation and Computational Linguistics. In Reed et al. 39], (to appear).

[18] F. Grasso, A. Cawsey, and R. Jones. Dialectical Argumentation to Solve Conflicts in Advice Giving: a case study in the promotion of healthy nutrition. International Journal of Human-Computer Studies, 53(6):1077-1115, 2000.

[19] N. Green. An Empirical Study of Multimedia Argumentation. In (current volume).

[20] R. Grishman. Computational Linguistics : an Introduction. Studies in Natural Language Processing. Cambridge University Press, 1986.

[21] L. Groarke. Logic, art and argument. Informal Logic, 18(2-3):105-129, 1996.

[22] B.J. Grosz and C.L Sidner. Plans for Discourse. In P. Cohen, J. Morgan, and M. Pollack, editors, Intentions in Communication, chapter 20, pages 417-444. MIT Press, Cambridge (Mass.), 1990.

[23] E. Hovy. Pragmatics and Natural Language Generation. Artificial Intelligence, 43:153-197, 1990.

[24] E. Hovy. Automated Discourse Generation using Discourse Structure Relations. Artificial Intelligence, 63(1-2):341-385, 1993.

[25] K. Jokinen, M. Maybury, M. Zock, and I. Zukerman, editors. Proceedings of the ECAI-96 Workshop on: Gaps and Bridges: New directions in Planning and NLG, 1996.

[26] N. Karacapilidis. An Argumentation Based Framework for Defeasible and Qualitative Reasoning. In Jokinen et al. 25], pages 37-42.

[27] W. Mann and S. Thompson. Rhetorical Structure Theory: Toward a Functional Theory of Text Organization. Text, 8(3):243-281, 1988.

[28] D. Marcu. The Conceptual and Linguistic Facets of Persuasive Arguments. In Jokinen et al. [25], pages 43-46.

[29] D. Marcu. The Theory and Practice of Discourse Parsing and Summarization. MIT Press, 2000.

[30] M. Maybury. Planning multimedia explanations using communicative acts. In Proceedings of the 9th National Conference on Artificial Intelligence (AAAI91), pages 61-66. AAAI, MIT press, July 1991. 
[31] M. Maybury. Communicative Acts for Generating Natural Language Arguments. In Proceedings of the 11th National Conference on Artificial Intelligence (AAA193), pages 357-364. AAAI, AAAI Press / The MIT Press, 1993.

[32] P. McBurney and S. Parsons. Agent ludens: Games for agent dialogues. In P. Gmytrasiewicz and S. Parsons, editors, Proceedings of the Third Workshop on Game-Theoretic and Decision-Theoretic Agents (GTDT2001), AAAI Spring Symposium. AAAI Press, Menlo Park, CA, 2001.

[33] K. McKeown. Text Generation: Using Discourse Strategy and Focus Constraints to Generate Natural Language Texts. Studies in Natural Language Processing. Cambridge University Press, 1985.

[34] J. Moore and C. Paris. Planning Text for Advisory Dialogues: Capturing Intentional and Rhetorical Information. Computational Linguistics, 19(4):651-695, 1993.

[35] C. Perelman and L. Olbrechts-Tyteca. The New Rhetoric: a treatise on argumentation. University of Notre Dame Press, Notre Dame, Indiana, 1969.

[36] J. Pollock. OSCAR: a General Theory of Rationality. In R. Cummins and J. Pollock, editors, Philosophy and AI: Essays at the Interface, chapter 9, pages 189-213. MIT Press, Cambridge (Mass.), 1991.

[37] C.A. Reed. The Role of Saliency in Generating Natural Language Arguments. In T. Dean, editor, Proceedings of the Sixteenth International Joint Conference on Artificial Intelligence (IJCAI'99), pages 876-881. Morgan Kaufmann Publishers, 1999.

[38] C.A. Reed and D.P. Long. Generating the structure of argument. In Proceedings of the 17th International Conference on Computational Linguistics and 36th Annual Meeting of the Association for Computational Linguistics (COLING-ACL'98), pages 1091-1097, 1998.

[39] C.A. Reed, T.J. Norman, and D. Gabbay, editors. Argument and Computation. (to appear).

[40] E. Reiter and R. Dale. Building Applied Natural Language Generation Systems. Natural Language Engineering, 3(1):57-87, 1997.

[41] S. Teufel, J. Carletta, and M. Moens. An Annotation Scheme for Discourse-Level Argumentation in Research Articles. In Proceedings of EACL, 1999.

[42] S. Toulmin. The Uses of Argument. Cambridge University Press, 1958.

[43] F. van Eemeren, R. Grootensdorst, F. Henkemans, J.A. Blair, R. Johnson, E. Krabbe, C. Plantin, D. Walton, C. Willard, J. Woods, and D. Zarefsky. Fundamentals of Argumentation Theory: A Handbook of Historical Backgrounds and Contemporary Developments. Lawrence Erlbaum Associates, Hillsdale, NJ, 1996.

[44] M.A. Walker. The Effect of Resource Limits and Task Complexity on Collaborative Planning in Dialogue. Artificial Intelligence, 85(1-2):181-243, 1996.

[45] P. Wason. Reasoning. In B.M. Foss, editor, New Horizons in Psychology. Harmondsworth: Penguin., 1966.

[46] B.A. Wilson. The Anatomy of Argument. University Press of America, 1980.

[47] I. Zukerman, K. Korb, and R. McConachy. Perambulations on the Way to an Architecture for a Nice Argument Generator. In Jokinen et al. [25], pages 32-36.

[48] I. Zukerman, R. McConachy, and K. Korb. Using Argumentation Strategies in Automated Argument Generation. In M. Elhadad, editor, Proceedings of the 1st International Conference on Natural Language Generation (INLG-2000), pages $55-62,2000$. 